\title{
Dynamic fracture analysis of an inclined subsurface crack subjected to dynamic moving loadings
}

\author{
CHIEN-CHING MA and LYANG-RUNG HWANG \\ Department of Mechanical Engineering, National Taiwan University, Taipei, Taiwan 10617, Republic of China
}

Received 28 July 1995; accepted in revised form 28 June 1996

\begin{abstract}
The transient response of a half-space containing a subsurface inclined semi-infinite crack excited by a dynamic moving antiplane loading on the surface of the half-space is investigated in this study. The solutions of dynamic stress intensity factors are derived for all load speeds (subsonic and supersonic) and are determined by superposition of a proposed fundamental solution in the Laplace transform domain. The fundamental problem is the problem of applying an exponentially distributed traction in the Laplace transform domain on crack faces. The method of analysis is based on integral transform techniques and the Wiener-Hopf technique. The exact closed form transient solutions of dynamic stress intensity factors are expressed in very compact formulations in this study. These solutions are valid for an infinite length of time and have accounted for all the contributions of infinitely many waves. Numerical results of the transient stress intensity factor are obtained and the results of the limit case of zero load speed is compared with the corresponding static values.
\end{abstract}

\section{Introduction}

A considerable amount of research has been directed towards the solution of problems involving the interaction of stress waves with cracks and boundaries to improve understanding of the behavior of material failure under dynamic loading. The study of crack interaction is also of great interest from the point of view of quantitative nondestructive evaluation of structural material. The scattered field carries information on the location and the size of the crack. There is considerable interest in scattering by cracks, with a view towards solving the inverse problem to obtain the crack geometry from the scattered fields.

Transient response due to a concentrated force moving in an infinite medium was determined by Payton [1] and the transient solution for a force moving in a half space was solved by Gakenheimer and Miklowitz [2]. In conventional studies of a semi-infinite crack in an unbounded medium subjected to a spatially uniform crack face traction, the complete solution is usually obtained by integral transform methods together with direct application of the Wiener-Hopf technique (Noble [3]) and the Cagniard-de Hoop method (de Hoop [4]) of Laplace inversion. If the crack face traction distribution is spatially nonuniform and a characteristic length is introduced, then this procedure using integral transformation methods does not apply. The problem of an elastic solid containing a semi-infinite crack subjected to concentrated impact loading on the faces of the crack has been studied by Freund [5]. He proposed a fundamental solution rising from an edge dislocation climbing along the line ahead of the crack tip with a constant speed to overcome these difficulties of the case with a characteristic length. The solution can be constructed by taking an integration over a climbing dislocation of different moving velocity. Basing their procedures on this method, Brock [6-8] and $\mathrm{Ma}$ and $\mathrm{Hou}[9,10]$ have analyzed a series of problems involving a semi-infinite crack subjected to dynamic impact loadings. 


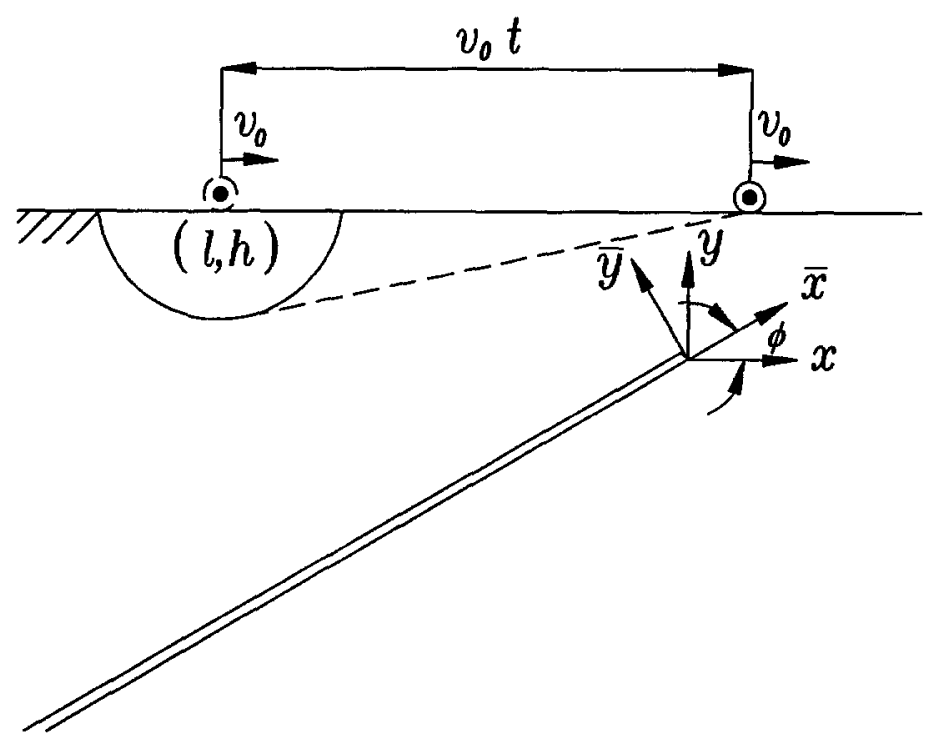

Figure 1. Configuration and coordinate systems of a subsurface inclined crack subjected to an impact loading moving with supersonic speed on the half-space.

Whenever dynamic loading is applied to a body with an internal crack, the resulting stress waves may initiate crack growth. Few analytical solutions for a cracked elastic solid subjected to dynamic loading are available. Exact transient closed form solutions for a stationary semiinfinite crack subjected to a suddenly applied dynamic body force in an unbounded medium have been obtained by Tsai and Ma [11] for the inplane case and by Ma and Chen [12] and Brock [13] for the antiplane case. The dynamic stress intensity factor for a half plane crack subjected to suddenly applied concentrated shear loads is obtained by Zhu and Kuang [14] by using the Laplace transforms together with the Wiener-Hopf technique and the Cagniardde Hoop method. All of the problems mentioned above are discussed for the case of a semi-infinite crack in an unbounded medium and the number of waves generated in those problems are all finite. The problem to be considered in this study is the transient response of an elastic half-plane with an inclined subsurface crack extending from infinity to a location near the half-plane surface. The cracked half plane is subjected to a dynamic antiplane loading moving with constant speed, either subsonic or supersonic, on the half-plane surface as shown in Figure 1. The most difficult part of this problem is that an infinite number of waves will be generated and all should be taken into account in the analysis. To the author's knowledge, most solutions for scattering of elastic waves by a crack, located in the immediate vicinity of a free surface, are given for incident plane time-harmonic waves (e.g. Achenbach and Brind [15] and Brind and Achenbach [16]). The only available transient analysis is presented by Ma and Chen [17] who consider the special case of applying dynamic loading with zero moving speed. The solution of the proposed transient problem in this study is complicated by the presence of the crack surface in addition to the free surface of the medium. The transient response of the cracked half plane is analyzed as the superposition of the fields in an uncracked half plane and the fields generated by appropriate surface tractions on the faces of the crack in the cracked half plane. In analyzing this problem, the reflections and diffractions of stress waves by the half-plane boundary and by the crack will generate an infinite number of waves that must be taken into account. This problem involves a characteristic length which makes a direct solution 
by standard techniques difficult. A new fundamental solution is used for overcoming these difficulties. This alternative fundamental solution is successfully applied towards solving the problem and is to be demonstrated as an efficient methodology. The final formulations for dynamic stress intensity factors are expressed explicitly and the dynamic effect of each wave is presented in a compact closed form. The results are valid for infinite waves that are scattered from the crack tip and reflected by the half-plane boundary. The corresponding result for the full field analysis has been obtained by $\mathrm{Ma}$ and Hwang [18]. Numerical results are presented for the loading, moving with various speeds along the half-plane free surface.

\section{The fundamental solutions}

Superposition of solutions plays a significant role in this study. The solutions of the problem considered in this study can be determined by superposition of the following problems. Problem A treats the dynamic concentrated force moving with constant velocity (subsonic and supersonic) on a half-plane medium without a crack, which induces a traction on the planes that will eventually define the semi-infinite crack faces. In problem $B$, an infinite body containing a semi-infinite crack is considered in which the crack faces are subjected to tractions which are equal and opposite to those on the corresponding planes in problem $\mathrm{A}$. Problem $\mathrm{C}$ considers a half-plane free surface subjected to the waves which are generated by the crack in problem B. Problem B in the above mentioned three fundamental problems is the only one which is difficult and needs careful analysis.

For most of the dynamic problems, the incident waves can be represented in an exponential functional form in the Laplace transform domain of time. The exponentially distributed loading acting on the crack faces can be analyzed by using the standard Wiener-Hopf technique, giving rise to a very useful result called the fundamental solution for the problem and the corresponding field quantities can be determined directly by superposition over this fundamental solution. The superposition scheme proposed in this study, unlike the usual superposition methods which are performed in the time domain, is performed in the Laplace transform domain.

The solution for applying an exponentially distributed loading on semi-infinite crack faces in the Laplace transform domain will be referred to as the fundamental solution. The fundamental problem can be viewed as a half-plane problem with the material occupying the region $y \geqslant 0$, subject to the following boundary conditions in the Laplace transform domain

$$
\begin{aligned}
& \bar{\tau}_{y z}(x, 0, p)=\mathrm{e}^{p \eta x}, \quad-\infty<x<0, \\
& \bar{w}(x, 0, p)=0, \quad 0<x<\infty,
\end{aligned}
$$

where $p$ is the Laplace transform parameter and $\eta$ is a constant. The overbar symbol is used for denoting the transform on time $t$. The governing equation can be represented by the following two-dimensional wave equation

$$
\frac{\partial^{2} w}{\partial x^{2}}+\frac{\partial^{2} w}{\partial y^{2}}=b^{2} \frac{\partial^{2} w}{\partial t^{2}},
$$

where $b$ is the slowness of the transverse wave given by

$$
b=1 / v_{s}=\sqrt{\rho / \mu},
$$


in which $w(x, y, t)$ is the displacement normal to the $x y$-plane; $v_{s}$ is the shear wave speed, $\mu$ and $\rho$ are the respective shear modulus and the mass density of the material. The nonvanishing shear stresses are

$$
\tau_{y z}=\mu \frac{\partial w}{\partial y}, \quad \tau_{x z}=\mu \frac{\partial w}{\partial x} .
$$

The above mentioned fundamental problem can be solved by the application of integral transforms. The solution is obtained by applying the one-sided Laplace transform over time, the two-sided Laplace transform over $x$ under the restriction of $\operatorname{Re}(\eta)>\operatorname{Re}(\lambda)$, and finally implementing the Wiener-Hopf technique. The fundamental solutions of stresses and displacement expressed in the Laplace transform domain for the boundary conditions (2.1) and (2.2) are

$$
\begin{aligned}
& \bar{\tau}_{y z}(x, y, p)=\frac{1}{2 \pi i} \int_{\Gamma_{\lambda}} \frac{(b+\lambda)^{1 / 2} \mathrm{e}^{-p(\alpha y-\lambda x)}}{(b+\eta)^{1 / 2}(\eta-\lambda)} \mathrm{d} \lambda, \\
& \bar{\tau}_{x z}(x, y, p)=-\frac{1}{2 \pi i} \int_{\Gamma_{\lambda}} \frac{\lambda \mathrm{e}^{-p(\alpha y-\lambda x)}}{(b+\eta)^{1 / 2}(\eta-\lambda)(b-\lambda)^{1 / 2}} \mathrm{~d} \lambda \\
& \bar{w}(x, y, p)=-\frac{1}{2 \pi i} \int_{\Gamma_{\lambda}} \frac{\mathrm{e}^{-p(\alpha y-\lambda x)}}{\mu p(b+\eta)^{1 / 2}(\eta-\lambda)(b-\lambda)^{1 / 2}} \mathrm{~d} \lambda
\end{aligned}
$$

where

$$
\alpha=\left(b^{2}-\lambda^{2}\right)^{1 / 2} .
$$

The fundamental solutions presented in (2.5)-(2.7) will be used to construct the reflected and diffracted waves generated by the crack in the following analysis. The corresponding result of the stress intensity factor expressed in the Laplace transform domain is

$$
\begin{aligned}
\bar{K}(p) & =\lim _{x \rightarrow 0} \sqrt{2 \pi x} \bar{\tau}_{y z}(x, 0, p) \\
& =-\frac{(2 / \pi)^{1 / 2} \Gamma(1 / 2)}{(b+\eta)^{1 / 2} p^{1 / 2}} .
\end{aligned}
$$

\section{Transient solutions of dynamic stress intensity factors}

Having two coordinate systems is convenient for the following analysis since the surface of the half-plane is not parallel to the crack faces. The origins of the two coordinate systems $(\bar{x}, \bar{y})$ and $(x, y)$ are both located at the crack tip as shown in Figure 1. The planar crack lies in the plane $\bar{y}=0, \bar{x}<0$ and the inclined angle of the crack is $\phi$. The valid range of $\phi$ for this analysis is $0 \leqslant \phi \leqslant \pi / 2$. The coordinate transforms and stress relations between these two systems are

$$
\begin{aligned}
& \bar{x}=x \cos \phi+y \sin \phi, \\
& \bar{y}=-x \sin \phi+y \cos \phi, \\
& \tau_{x z}=\tau_{\overline{x z}} \cos \phi-\tau_{\overline{y z}} \sin \phi,
\end{aligned}
$$




$$
\tau_{y z}=\tau_{\overline{x z}} \sin \phi+\tau_{\overline{y z}} \cos \phi .
$$

The investigation of a subsurface crack subjected to dynamic moving loading is an important topic in dynamic fracture analysis. The problem considered here is an inclined semiinfinite crack located under the surface of a half-plane as shown in Figure 1. The problem has a characteristic length and a direct attempt towards solving this problem by transform and Wiener-Hopf techniques is not applicable. The transient elastodynamic problem will be solved by superposition of the fundamental solutions obtained in the previous section in the Laplace transform domain. The transient solutions are composed of an incident field, reflected field, and diffracted field, which will be denoted by superscripts of $i, r$, and $d$ for the case of subsonic moving speed and additional superscripts of $I, R$ and $D$ will be used for the supersonic case, respectively. The incident wave is the response for applying a dynamic loading that moves along an uncracked half-plane with constant velocity. The reflected and diffracted waves are generated through application of an opposite traction at the crack surface thus eliminating the stress induced by the incident wave.

Consider a half-plane medium which is stress-free and at rest. At time $t=0$, an antiplane concentrated dynamic load of magnitude $Q$ is applied at the free surface of a halfplane at position $(l, h)$. For $t>0$, this concentrated load travels on the surface along the positive $x$-axis with a constant speed $v_{0}$. The time dependence of the loading is represented by the Heaviside step function $H(t)$. The boundary condition on the half-plane surface is $\tau_{y z}(x, h, t)=Q \delta\left(x-l-v_{0} t\right) H(t)$. The incident fields of shear stresses in the Laplace transform domain can be represented as follows

$$
\begin{aligned}
& \bar{\tau}_{y z}^{i}(x, y, p)=\frac{1}{2 \pi i} \int_{\Gamma_{\lambda}} \frac{Q}{1+\lambda v_{0}} \mathrm{e}^{p[\alpha(y-h)+\lambda(x-l)]} \mathrm{d} \lambda, \\
& \bar{\tau}_{x z}^{i}(x, y, p)=\frac{1}{2 \pi i} \int_{\Gamma_{\lambda}} \frac{\lambda Q}{\alpha\left(1+\lambda v_{0}\right)} \mathrm{e}^{p[\alpha(y-h)+\lambda(x-l)]} \mathrm{d} \lambda .
\end{aligned}
$$

The Laplace transform is inverted by a modification of Cagniard's [19] technique due to de Hoop [4]. For this technique (3.5) and (3.6) are converted into the Laplace transform of a known function, so that the Laplace transform can be inverted by inspection. In the subsequent calculations $p$ is assumed to be a real and positive number. The transient solution has different forms depending on the speed of the moving load relative to the shear wave speed. In particular, the terms supersonic and subsonic refer to the cases when the load speed is greater than the shear wave speed $\left(v_{0}>v_{s}\right)$ or less than the shear wave speed $\left(v_{0}<v_{s}\right)$, respectively.

The full field transient solutions of incident wave in time domain can be obtained as follows

$$
\begin{aligned}
\tau_{y z}^{i}(x, y, t)= & \frac{Q\left(-v_{0} b^{2} r^{2} \sin \theta \cos \theta+r t \sin \theta\right)}{\pi\left(t^{2}-b^{2} r^{2}\right)^{1 / 2}\left(r^{2}-2 r v_{0} t \cos \theta-v_{0}^{2} b^{2} r^{2} \sin ^{2} \theta+v_{0}^{2} t^{2}\right)} H(t-b r) \\
& +\frac{Q}{v_{0}} \delta\left[t-c(x-l)+\sqrt{b^{2}-c^{2}}(y-h)\right] H\left(v_{0}-v_{s}\right) H(b \cos \theta-c), \\
\tau_{x z}^{i}(x, y, t)= & \frac{Q\left(v_{0} t^{2}-v_{0} b^{2} r^{2} \sin ^{2} \theta-r t \cos \theta\right)}{\pi\left(t^{2}-b^{2} r^{2}\right)^{1 / 2}\left(r^{2}-2 r v_{0} t \cos \theta-v_{0}^{2} b^{2} r^{2} \sin ^{2} \theta+v_{0}^{2} t^{2}\right)} H(t-b r) \\
& -\frac{Q}{v_{0} \sqrt{b^{2} v_{0}^{2}-1}} \delta\left[t-c(x-l)+\sqrt{b^{2}-c^{2}}(y-h)\right] \\
& \times H\left(v_{0}-v_{s}\right) H(b \cos \theta-c),
\end{aligned}
$$


where

$$
r=\left[(x-l)^{2}+(y-h)^{2}\right]^{1 / 2}, \quad \theta=\cos ^{-1}\left(\frac{x-l}{r}\right),
$$

and $c=1 / v_{0}$ is the slowness of the speed of the moving load. The symbols $\delta(\cdot)$ and $H(\cdot)$ represent the Dirac delta function and the unit step function, respectively. The contribution of the second term shown in (3.7)-(3.8) only exists for the supersonic case and represents a plane wave. For the subsonic case, only the first term is left in the transient solution expressed in (3.7)-(3.8).

The incident wave will induce a stress field $\bar{\tau} \frac{i}{y z}(\bar{x}, 0, p)$ along the semi-infinite crack face, conveniently expressed in the $\bar{x}-\bar{y}$ coordinate system as

$$
\bar{\tau} \frac{i}{y z}(\bar{x}, 0, p)=\frac{1}{2 \pi i} \int_{\Gamma_{\lambda}} \frac{Q}{\left(1+\lambda v_{o} \cos \phi-\alpha v_{0} \sin \phi\right)} \mathrm{e}^{-p \alpha h_{0}+p \lambda\left(\bar{x}-l_{0}\right)} \mathrm{d} \lambda,
$$

where

$$
l_{0}=l \cos \phi+h \sin \phi, \quad h_{0}=-l \sin \phi+h \cos \phi .
$$

The applied traction on the crack face, in order to eliminate the stress induced by the incident wave as indicated in (3.9), has the functional form $\mathrm{e}^{p \lambda \bar{x}}$. Since the solutions of applying traction $\mathrm{e}^{p \eta \bar{x}}$ on crack faces have been solved in Section 2 , the reflected and diffracted fields generated from the inclined crack can be constructed by superimposing the incident wave traction that is equal and opposite to (3.9). When we combine (2.5) and (3.9), the solution of the first reflected and diffracted waves for $\bar{\tau} \overline{y z}$ and $\bar{\tau} \overline{x z}$ in the Laplace transform domain can be expressed as follows

$$
\begin{aligned}
\bar{\tau}_{\overline{y z}}^{d+r}(\bar{x}, \bar{y}, p)= & \frac{Q}{4 \pi^{2}} \int_{\Gamma_{\eta_{1}}} \int_{\Gamma_{\eta_{2}}} \frac{G\left(\eta_{1}, \eta_{2}\right)}{\left[1+v_{0}\left(\eta_{1} \cos \phi-\alpha_{1} \sin \phi\right)\right]} \\
& \times \mathrm{e}^{-p\left(\alpha_{1} h_{0}+\eta_{1} l_{0}\right)} \mathrm{e}^{-p\left(\alpha_{2} \bar{y}-\eta_{2} \bar{x}\right)} \mathrm{d} \eta_{2} \mathrm{~d} \eta_{1}, \\
\bar{\tau}_{\overline{x z}}^{d+r}(\bar{x}, \bar{y}, p)= & -\frac{Q}{4 \pi^{2}} \int_{\Gamma_{\eta_{1}}} \int_{\Gamma_{\eta_{2}}} \frac{\eta_{2}}{\alpha_{2}} \frac{G\left(\eta_{1}, \eta_{2}\right)}{\left[1+v_{0}\left(\eta_{1} \cos \phi-\alpha_{1} \sin \phi\right)\right]} \\
& \times \mathrm{e}^{-p\left(\alpha_{1} h_{0}+\eta_{1} l_{0}\right)} \mathrm{e}^{-p\left(\alpha_{2} \bar{y}-\eta_{2} \bar{x}\right)} \mathrm{d} \eta_{2} \mathrm{~d} \eta_{1},
\end{aligned}
$$

where

$$
\begin{aligned}
& G\left(\eta_{1}, \eta_{2}\right)=\frac{\left(b+\eta_{2}\right)^{1 / 2}}{\left(b+\eta_{1}\right)^{1 / 2}\left(\eta_{1}-\eta_{2}\right)} \\
& \alpha_{1}=\left(b^{2}-\eta_{1}^{2}\right)^{1 / 2}, \quad \alpha_{2}=\left(b^{2}-\eta_{2}^{2}\right)^{1 / 2} .
\end{aligned}
$$

Since the stress intensity factor of applying traction $\mathrm{e}^{p \eta \bar{x}}$ has been obtained in the previous section, the solution of the dynamic stress intensity factor due to the incident wave in the Laplace transform domain can be represented as follows

$$
\bar{K}(p)=\frac{1}{2 \pi i} \int_{\Gamma_{\eta_{1}}} \frac{Q(2 / \pi)^{1 / 2} \Gamma(1 / 2)}{\left[1+v_{0}\left(\eta_{1} \cos \phi-\alpha_{1} \sin \phi\right)\right]\left(b+\eta_{1}\right)^{1 / 2} p^{1 / 2}} \mathrm{e}^{-p\left(\alpha_{1} h_{0}+\eta_{1} l_{0}\right)} \mathrm{d} \eta_{1} .
$$


By using the Cagniard-de Hoop method of the Laplace inversion, the dynamic stress intensity factor in time domain can be obtained by Cagniard-de Hoop method. The idea of the Cagniard-de Hoop method is to deform the path of integration in the complex $\eta_{1}$-plane in such a manner that the inverse Laplace transform of the integral along the new path of integration can be obtained by inspection. From the following elementary property of the one-sided Laplace transform

$$
\mathcal{L}^{-1}\left\{\int_{t_{1}}^{\infty} \mathrm{e}^{-p t} g(t) \mathrm{d} t\right\}=g(t) H\left(t-t_{1}\right)
$$

the inversion of the two transforms (i.e., the transform in time $t$ and in the spatial variable $x$ ) can be operated at one time. We consider a path of integration in the $\eta_{1}$-plane defined by

$$
\alpha_{1} h_{0}+\eta_{1} l_{0}=t
$$

where $t$ is real and positive. This equation can be solved for $\eta_{1}$ to yield

$$
\eta_{1}^{ \pm}=\frac{t \cos \Theta_{0}}{R_{0}} \pm i \frac{\sin \Theta_{0}}{R_{0}}\left(t^{2}-b^{2} R_{0}^{2}\right)^{1 / 2},
$$

where

$$
R_{0}=\left(l_{0}^{2}+h_{0}^{2}\right)^{1 / 2}, \quad \Theta_{0}=\cos ^{-1}\left(l_{0} / R_{0}\right) .
$$

This represents essentially a transformation of the $t$-plane to the $\eta_{1}$-plane which changes the path of integration $\Gamma_{\eta_{1}}$ to $\Gamma_{\eta_{1}^{ \pm}}$. The inversion of the Laplace transform of (3.11) in the time domain is

$$
\begin{aligned}
K(t)= & \sqrt{\frac{2}{\pi^{3}}} \int_{b R_{0}}^{t} \operatorname{Im}\left[\frac{Q \partial \eta_{1}^{+} / \partial \xi}{\left[1+v_{0}\left(\eta_{1}^{+} \cos \phi-\alpha_{1}^{+} \sin \phi\right)\right]\left(b+\eta_{1}^{+}\right)^{1 / 2}}\right] \frac{1}{\sqrt{t-\xi}} \mathrm{d} \xi \\
& +Q \sqrt{\frac{2}{\pi}} \frac{\left(\sqrt{b^{2} v_{0}^{2}-1} \cos \phi+\sin \phi\right)\left(t-\sqrt{b^{2}-c^{2}} h+c l\right)^{-1 / 2}}{\sqrt{b^{2} v_{0}^{2}-1}\left(b+\sqrt{b^{2}-c^{2}} \sin \phi-c \cos \phi\right)} \\
& \times H\left(v_{0}-v_{s}\right) H\left(t-\sqrt{b^{2}-c^{2}} h+c l\right),
\end{aligned}
$$

where

$$
\alpha_{1}^{+}=\left[b^{2}-\left(\eta_{1}^{+}\right)^{2}\right]^{1 / 2}
$$

For the supersonic case $\left(v_{0}>v_{s}\right)$, the incident wave contains an additional plane wave and this plane wave will also make a contribution to the stress intensity factor which is represented in the second term in (3.12). The configuration of the wave fronts for the supersonic case is shown in Figure 2. The reflected wave ( $R$ wave) and diffracted wave ( $D$ wave) generated from the crack by the incident plane wave are constructed from the contribution of the pole (i.e. $1+v_{0}\left(\eta_{1} \cos \phi-\alpha_{1} \sin \phi\right)=0$ ) shown in (3.11), which is $\eta_{1}=-c \cos \phi+\sqrt{b^{2}-c^{2}} \sin \phi$. Hence, the second term in (3.12) is obtained from the contribution from this pole for the inverse Laplace transform of (3.11).

For the subsonic case, the incident cylindrical shear wave ( $i$ wave) will generate only a reflected wave ( $r$ wave) and a diffracted wave ( $d$ wave) from the subsurface crack. After some later time, these two waves will be reflected from the free half-surface and are indicated as the 


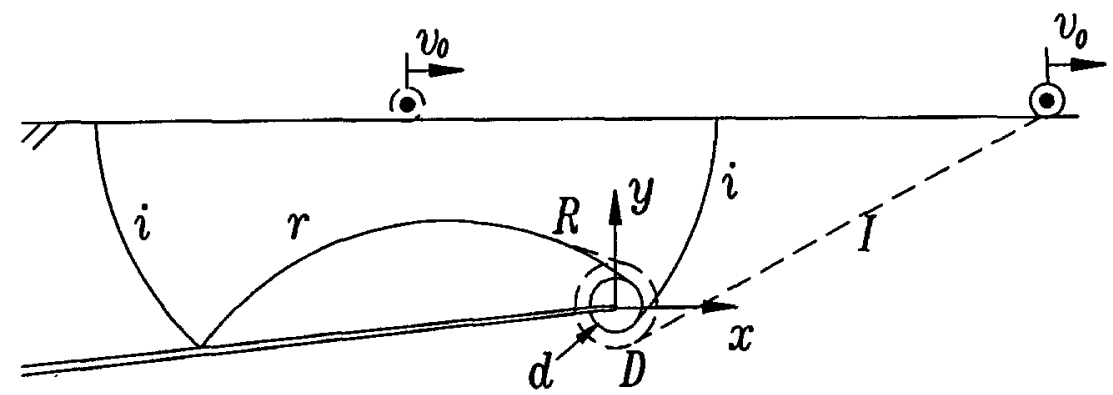

Figure 2. Wave fronts for reflected and diffracted waves generated from the inclined crack by incident plane and cylindrical waves.

$r r$ wave and $d r$ wave. The solutions for $r r$ and $d r$ waves can be constructed by employing the method of images, which can be obtained from the solutions of $r$ and $d$ waves, and the results are omitted here. The reflected $r r$ and $d r$ waves will arrive at the crack at a later time. The traction induced on the crack face by $r r$ and $d r$ waves in the Laplace transform domain can be expressed as follows

$$
\begin{aligned}
\bar{\tau}_{\overline{y z}}(\bar{x}, 0, p)= & \frac{Q}{4 \pi^{2}} \int_{\Gamma_{\eta_{1}}} \int_{\Gamma_{S_{22}}} F\left(\eta_{1}, \eta_{2}\right) \mathrm{e}^{-p\left(\alpha_{1} h_{0}+\eta_{1} l_{0}\right)} \\
& \times \mathrm{e}^{-2 p h\left(\beta_{22} \cos \phi+S_{22} \sin \phi\right)} \mathrm{e}^{p S_{22} \bar{x}} \mathrm{~d} S_{22} \mathrm{~d} \eta_{1},
\end{aligned}
$$

where

$$
\begin{aligned}
& F\left(\eta_{1}, \eta_{2}\right)=\frac{G\left(\eta_{1}, \eta_{2}\right)}{1+v_{0}\left(\eta_{1} \cos \phi-\alpha_{1} \sin \phi\right)}, \\
& S_{12}=\alpha_{2} \sin \phi+\eta_{2} \cos \phi, \quad \beta_{12}=\alpha_{2} \cos \phi-\eta_{2} \sin \phi \\
& S_{22}=\beta_{12} \sin \phi+S_{12} \cos \phi=\alpha_{2} \sin 2 \phi+\eta_{2} \cos 2 \phi, \\
& \beta_{22}=\left(b^{2}-S_{22}^{2}\right)^{1 / 2}=\alpha_{2} \cos 2 \phi-\eta_{2} \sin 2 \phi .
\end{aligned}
$$

The applied traction on the crack face has the functional form of $\mathrm{e}^{p S_{22} \bar{x}}$ as shown in (3.13). Since the solutions of applying traction $\mathrm{e}^{p \eta \bar{x}}$ on the crack face have been previously solved in Section 2, the dynamic stress intensity of the inclined crack due to the contribution for incident $r r$ and $r d$ waves can be constructed by superimposing (2.8) and (3.13). The solution for the dynamic stress intensity factor expressed in the Laplace transform domain is

$$
\begin{aligned}
\bar{K}(p)= & -\frac{Q \sqrt{2 / \pi}}{4 \pi^{2}} \int_{\Gamma_{\eta_{1}}} \int_{\Gamma_{S_{22}}} \frac{F\left(\eta_{1}, \eta_{2}\right) \Gamma(1 / 2)}{p^{1 / 2}\left(b+S_{22}\right)^{1 / 2}} \mathrm{e}^{-p\left(\alpha_{1} h_{0}+\eta_{1} l_{0}\right)} \\
& \times \mathrm{e}^{-2 p h\left(\beta_{22} \cos \phi+S_{22} \sin \phi\right)} \mathrm{d} S_{22} \mathrm{~d} \eta_{1} .
\end{aligned}
$$

A similar procedure is followed as previously indicated for constructing the contribution from the incident wave. Cagniard contours are introduced here by setting

$$
\begin{aligned}
& \alpha_{1} h_{0}+\eta_{1} l_{0}=t_{1}, \\
& 2 h\left(\beta_{22} \cos \phi+S_{22} \sin \phi\right)=t_{2},
\end{aligned}
$$


which can be solved for $\eta_{1}$ and $S_{22}$ as follows

$$
\begin{gathered}
\eta_{1}^{ \pm}=\frac{t_{1} \cos \Theta_{0}}{R_{0}} \pm i \frac{\sin \Theta_{0}}{R_{0}}\left(t_{1}^{2}-b^{2} R_{0}^{2}\right)^{1 / 2}, \\
S_{22}^{ \pm}=\frac{t_{2} \sin \phi}{2 h} \pm i \frac{\cos \phi}{2 h}\left(t_{2}^{2}-4 b^{2} h^{2}\right)^{1 / 2} .
\end{gathered}
$$

We shift the $\eta_{1}$ - and $S_{22}$-integration onto the associated Cagniard contours along which $t_{1}$ and $t_{2}$ are both real and positive. The two Cagniard contours must be superimposed in this technique for different locations of loading point and crack tip. Because $F\left(\eta_{1}, \eta_{2}\right)$ possesses a pole at $\eta_{1}=\eta_{2}$, the contribution of the pole has to be taken into account in the change of integral paths from $\eta_{1}$ to $t_{1}$ and $\eta_{2}$ to $t_{2}$. The contribution of this pole represents the fact that the reflected $r r$ wave would pass the crack tip and the condition for this to happen is $\Theta_{0}>\frac{1}{2} \pi+\phi$. Hence the contribution of the dynamic stress intensity factor due to $r r$ and $d r$ waves in time domain can be expressed as follows

$$
\begin{aligned}
K(t)= & \sqrt{\frac{2}{\pi^{3}}} \int_{b R_{02}}^{t} \operatorname{Im}\left[\frac{Q \partial T_{22}^{+} / \partial \xi}{\left[1+v_{0}\left(\Lambda_{2}^{+} \cos \phi-P_{2}^{+} \sin \phi\right)\right]\left(b+T_{22}^{+}\right)^{1 / 2}}\right] \frac{1}{\sqrt{t-\xi}} \mathrm{d} \xi \\
& -\frac{\sqrt{2 / \pi}}{2 \pi^{2}} \int_{b R_{0}+2 b h}^{t} \frac{1}{(t-u)^{1 / 2}} \int_{b R_{0}}^{u-2 b h} \operatorname{Re}\left[\frac{F\left(\eta_{1}^{+}, \eta_{2}^{+}\right)}{\left(b+S_{22}^{+}\right)^{1 / 2}} \frac{\partial \eta_{1}^{+}}{\partial t_{1}} \frac{\partial S_{22}^{+}}{\partial t_{2}}\right. \\
& \left.-\frac{F\left(\eta_{1}^{-}, \eta_{2}^{+}\right)}{\left(b+S_{22}^{+}\right)^{1 / 2}} \frac{\partial \eta_{1}^{-}}{\partial t_{1}} \frac{\partial S_{22}^{+}}{\partial t_{2}} \mathrm{~d} t_{1}\right] \mathrm{d} u \quad \text { for } \Theta_{0}>\frac{1}{2} \pi+\phi, \\
K(t)= & -\frac{\sqrt{2 / \pi}}{2 \pi^{2}} \int_{b R_{0}+2 b h}^{t} \frac{1}{(t-u)^{1 / 2}} \int_{b R_{0}}^{u-2 b h} \operatorname{Re}\left[\frac{F\left(\eta_{1}^{+}, \eta_{2}^{+}\right)}{\left(b+S_{22}^{+}\right)^{1 / 2}} \frac{\partial \eta_{1}^{+}}{\partial t_{1}} \frac{\partial S_{22}^{+}}{\partial t_{2}}\right. \\
& \left.-\frac{F\left(\eta_{1}^{-}, \eta_{2}^{+}\right)}{\left(b+S_{22}^{+}\right)^{1 / 2}} \frac{\partial \eta_{1}^{-}}{\partial t_{1}} \frac{\partial S_{22}^{+}}{\partial t_{2}} \mathrm{~d} t_{1}\right] \mathrm{d} u \quad \text { for } \Theta_{0}>\frac{1}{2} \pi+\phi,
\end{aligned}
$$

where

$$
\begin{aligned}
& T_{22}^{+}=\frac{\xi \cos \Theta_{02}}{R_{02}}+i \frac{\sin \Theta_{02}}{R_{02}}\left(\xi^{2}-b^{2} R_{02}^{2}\right)^{1 / 2}, \\
& \Lambda_{2}^{+}=T_{22}^{+} \cos 2 \phi-\left[b^{2}-\left(T_{22}^{+}\right)^{2}\right]^{1 / 2} \sin 2 \phi, \\
& P_{2}^{+}=\left[b^{2}-\left(T_{22}^{+}\right)^{2}\right]^{1 / 2} \cos 2 \phi+T_{22}^{+} \sin 2 \phi, \\
& R_{02}=\left(l_{02}^{2}+h_{01}^{2}\right)^{1 / 2}, \quad \Theta_{02}=\cos ^{-1}\left(\frac{l_{02}}{R_{02}}\right), \\
& l_{02}=l \cos 3 \phi+h \sin 3 \phi+2 h \sin \phi, \quad h_{02}=-l \sin 3 \phi+h \cos 3 \phi+2 h \cos \phi, \\
& t_{2}=t-t_{1} .
\end{aligned}
$$

The arrival times of the wave fronts for $r r$ and $d r$ waves are found to be $b R_{02}$ and $b\left(2 h+R_{0}\right)$, respectively. The contributions of the $r r$ wave and $d r$ wave for the dynamic stress intensity factor are expressed in the first term and second term of (3.15), respectively. 


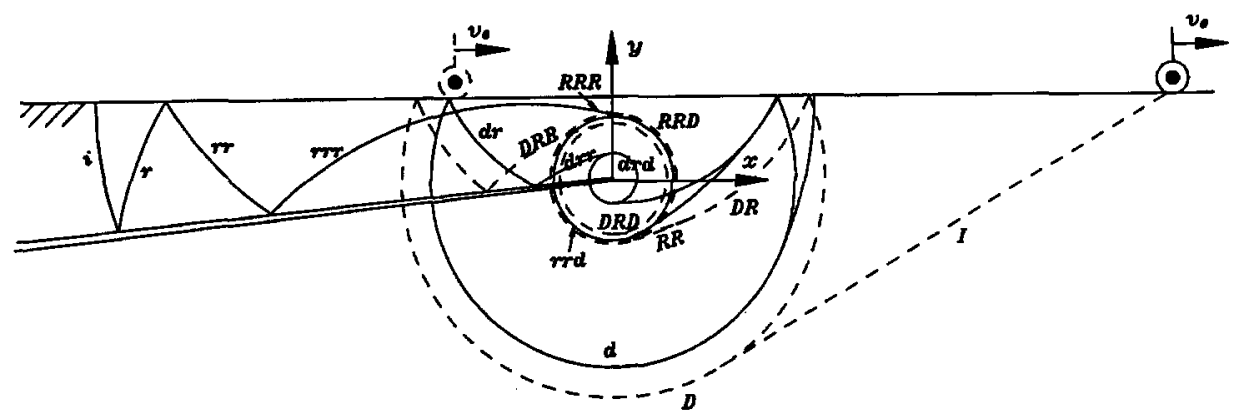

Figure 3. Wave fronts of the incident, reflected and diffracted waves for a short time period after the impact loading is applied on the half-space for the supersonic case.

For the supersonic case, additional waves should be included which are initiated by the incident plane wave. There are two waves, i.e. $D R$ and $R R$ waves, the explicit expressions of stress intensity factor due to the contributions of these two waves are

$$
\begin{aligned}
K(t)= & \frac{2 Q}{\pi^{3 / 2}}\left[\frac{\left(\sqrt{b^{2} v_{0}^{2}-1} \cos \phi+\sin \phi\right)}{\sqrt{b^{2} v_{0}^{2}-1}\left(b-c \cos \phi+\sqrt{b^{2}-c^{2}} \sin \phi\right)^{1 / 2}}\right] \\
& \times \int_{0}^{t} \frac{1}{\sqrt{t-u}} \operatorname{Im}\left[\frac{\left(b+\eta_{2}^{+}\right)^{1 / 2}}{\left(-\cos \phi+\sqrt{b^{2} v_{0}^{2}-1} \sin \phi-\eta_{2}^{+} v_{0}\right)\left(b+\zeta_{2}^{+}\right)^{1 / 2}} \frac{\partial \zeta_{2}^{+}}{\partial u}\right] \\
& \times H\left(u-\sqrt{b^{2}-c^{2}} h+c l-2 b h\right) \mathrm{d} u \\
K(t)= & \sqrt{\frac{2}{\pi}} \frac{Q\left(\sqrt{b^{2} v_{0}^{2}-1} \cos 3 \phi+\sin 3 \phi\right)}{\sqrt{b^{2} v_{0}^{2}-1}\left(b-c \cos 3 \phi+\sqrt{b^{2}-c^{2}} \sin 3 \phi\right)^{1 / 2}} \\
& \times \frac{H\left[t-\sqrt{b^{2}-c^{2}}(h+2 h \cos 2 \phi)+c(l-2 h \sin 2 \phi)\right.}{\left[t-\sqrt{b^{2}-c^{2}}(h+2 h \cos 2 \phi)+c(l-2 h \sin 2 \phi)\right]^{-1 / 2}},
\end{aligned}
$$

where

$$
\zeta_{2}^{+}=\frac{\sin \phi}{2 h}\left[u-\sqrt{b^{2}-c^{2}} h+c l\right]+i \frac{\cos \phi}{2 h}\left[\left(u-\sqrt{b^{2}-c^{2}} h+c l\right)^{2}-4 b^{2} h^{2}\right]^{1 / 2} .
$$

The correspondent configuration of the wave fronts for the supersonic case is shown in Figure 3. Following a similar procedure to that used for constructing the dynamic stress intensity factor for the first few waves, the complete transient solution of the dynamic stress intensity factor is finally obtained explicitly. The solutions of all the contributions for reflected and diffracted waves are included. The complete solution for dynamic stress intensity factor can be simplified into a very compact form as follows

$$
\begin{aligned}
K(t)= & \sum_{k=0}^{n}\left[K^{k}(t)+\sum_{l=1}^{\infty} K^{k, l}(t)\right] \\
& +\sum_{k^{\prime}=0}^{n^{\prime}}\left[K^{k^{\prime}}(t)+\sum_{l^{\prime}=1}^{\infty} K^{k^{\prime}, l^{\prime}}(t)\right] H\left(v_{0}-v_{s}\right) .
\end{aligned}
$$


For the subsonic case, the complete solutions only consist of the first two terms in (3.19). The first term with one summation is the contributions for incident wave and reflected waves which are only generated by purely reflected waves, i.e., $k=0$ for incident wave, $k=1$ for $r r$ wave, $k=2$ for $r r r r$ wave. The explicit forms are expressed as follows

$$
\begin{aligned}
K^{k}(t)= & \sqrt{\frac{2}{\pi^{3}}} \int_{b R_{0,2 k}}^{t} \\
& \times \operatorname{Im}\left[\frac{\partial S_{2, k+1}^{+} / \partial \tau}{\left[1+v_{0}\left(\eta_{k+1}^{+} \cos \phi-\alpha_{k+1}^{+} \sin \phi\right)\right]\left(b+S_{2, k+1}^{+}\right)^{1 / 2}}\right] \frac{1}{\sqrt{t-\tau}} \mathrm{d} \tau
\end{aligned}
$$

where

$$
\begin{aligned}
& S_{2, k+1}^{+}=\frac{\tau \cos \Theta_{0,2 k}+i \sin \Theta_{0,2 k}\left(\tau^{2}-b^{2} R_{0,2 k}^{2}\right)^{1 / 2}}{R_{0,2 k}}, \\
& \eta_{k+1}^{+}=S_{2, k+1}^{+} \cos 2 \phi-\beta_{2, k+1}^{+} \sin 2 \phi, \\
& R_{0,2 k}=\left(l_{0,2 k}^{2}+h_{0,2 k}^{2}\right)^{1 / 2}, \quad \cos \Theta_{0,2 k}=\frac{l_{0,2 k}}{R_{0,2 k}}, \\
& l_{0,2 k}=l \cos (2 k+1) \phi+h \sin (2 k+1) \phi+\sum_{m=0}^{k-1} 2 h \sin (2 m+1) \phi, \\
& h_{0,2 k}=-l \sin (2 k+1) \phi+h \cos (2 k+1) \phi+\sum_{m=0}^{k-1} 2 h \cos (2 m+1) \phi .
\end{aligned}
$$

The second term in (3.19) with two summations comes from the contributions for reflected waves from the half-plane surface which are generated by diffracted waves from the crack tip, i.e., $k=0, l=1$ for $d r$ wave, $l=2$ for $d r d r$ wave, $l=3$ for $d r d r d r$ wave; $k=1, l=1$ for $r r d r$ wave, $l=2$ for $r r d r d r$ wave; $l=3$ for $r r d r d r d r$ wave. $k=0$ can be seen here to consist of a diffracted $d$ wave which is diffracted by the incident wave and an infinite sequence of reflected waves (i.e., $d r$ wave, $d r d r$ wave, etc.). $k=1$ consists of diffracted $r r d$ wave which is diffracted by the crack tip for the $r r$ wave and an infinite sequence of reflected waves (i.e., $r r d r$ wave, $r r d r d r$ wave, etc.). The results of the dynamic stress intensity factor due to the contribution of each wave indicated above can be expressed as follows

$$
\begin{aligned}
K^{k, l}(t)= & \frac{-\sqrt{2 / \pi}(i)^{q}}{2 \pi^{2}(2 \pi i)^{l-1}} \int_{b R_{0,2 k}+2 l h b}^{t} \frac{1}{(t-u)^{1 / 2}} \int_{b R_{0,2 k}}^{b_{1}} \int_{2 h b}^{b_{2}} \ldots \\
& \cdots \int_{2 h b}^{b_{l}} F U N K_{k, l} \mathrm{~d} t_{l} \mathrm{~d} t_{l-1}, \ldots, \mathrm{d} t_{1} \mathrm{~d} u
\end{aligned}
$$

where

$$
F U N K_{k, l}=\operatorname{Re}\left[\frac{G\left(S_{2, k+1}^{ \pm}, \eta_{k+2}^{ \pm}\right) G\left(S_{2, k+2}^{ \pm}, \eta_{k+3}^{ \pm}\right) \cdots}{\left[1+v_{0}\left(\eta_{k+1}^{ \pm} \cos \phi-\alpha_{k+1}^{ \pm} \sin \phi\right)\right]\left(b+S_{2, k+l+1}^{+}\right)^{1 / 2}}\right.
$$




$$
\begin{aligned}
\times & G\left(S_{2, k+l}^{ \pm}, \eta_{k+l+1}^{+}\right)\left( \pm \frac{\partial S_{2, k+1}^{ \pm}}{\partial t_{k+1}}\right)\left( \pm \frac{\partial S_{2, k+2}^{ \pm}}{\partial t_{k+2}}\right) \cdots \\
& \left.\cdots\left( \pm \frac{\partial S_{2, k+l}^{ \pm}}{\partial t_{k+l}}\right)\left(\frac{\partial S_{2, k+l+1}^{+}}{\partial t_{k+l+1}}\right)\right] \text { for } l=1,3,5, \ldots \\
F U N K_{k, l}= & \operatorname{Im}\left[\frac{G\left(S_{2, k+1}^{ \pm}, \eta_{k+2}^{ \pm}\right) G\left(S_{2, k+2}^{ \pm}, \eta_{k+3}^{ \pm}\right) \cdots}{\left[1+v_{0}\left(\eta_{k+1}^{ \pm} \cos \phi-\alpha_{k+1}^{ \pm} \sin \phi\right)\right]\left(b+S_{2, k+l+1}^{+}\right)^{1 / 2}}\right. \\
& \times G\left(S_{2, k+l}^{ \pm}, \eta_{k+l+1}^{+}\right)\left( \pm \frac{\partial S_{2, k+1}^{ \pm}}{\partial t_{k+1}}\right)\left( \pm \frac{\partial S_{2, k+2}^{ \pm}}{\partial t_{k+2}}\right) \cdots \\
& \left.\cdots\left( \pm \frac{\partial S_{2, k+l}^{ \pm}}{\partial t_{k+l}}\right)\left(\frac{\partial S_{2, k+l+1}^{+}}{\partial t_{k+l+1}}\right)\right] \quad \text { for } l=2,4,6, \ldots
\end{aligned}
$$

in which

$$
\begin{aligned}
& S_{2, k+1}^{ \pm}=-\frac{t_{k+1} \cos \Theta_{0,2 k}}{R_{0,2 k}} \pm i \frac{\sin \Theta_{0,2 k}}{R_{0,2 k}}\left(t_{k+1}^{2}-b^{2} R_{0,2 k}^{2}\right)^{1 / 2}, \\
& S_{2, k+v+1}^{ \pm}=\frac{t_{k+v+1} \sin \phi}{2 h} \pm i \frac{\cos \phi}{2 h}\left(t_{k+v+1}^{2}-4 b^{2} h^{2}\right)^{1 / 2}, \quad v=1,2,3, \ldots, l, \\
& \eta_{k+v+1}^{ \pm}=-\frac{t_{k+v+1} \sin \phi}{2 h} \pm i \frac{\cos \phi}{2 h}\left(t_{k+v+1}^{2}-4 b^{2} h^{2}\right)^{1 / 2}, \quad v=1,2,3, \ldots, l \\
& q=1, \text { when } l=2,4,6, \ldots ; \quad q=0, \text { when } l=1,3,5,7, \ldots, \\
& b_{1}=u-2 l h b, b_{m+1}=u-t_{1}-t_{2}, \ldots,-t_{m}-2(l-m) h b, \quad m=1,2, \ldots, l-1 .
\end{aligned}
$$

However, for the supersonic case $\left(v_{0}>v_{s}\right)$, the incident waves generated by the moving loading consist of not only a cylindrical wave but also a plane wave as shown in Figure 1. We have already expressed the complete expressions of the dynamic stress intensity factor induced by an incident cylindrical wave in (3.20)-(3.21). The reflected and diffracted waves generated by the incident plane wave are expressed in (3.19) for the last two terms. The term with one summation is the solution of incident plane wave and reflected waves which are generated by pure reflected waves, i.e., $k^{\prime}=0$ for incident plane wave, $k^{\prime}=1$ for $R$ wave, $k^{\prime}=2$ for $R R$ wave, $k^{\prime}=3$ for $R R R$ wave and all these waves are plane waves. The explicit form of the dynamic stress intensity factors due to the contributions of these plane waves can be expressed as follows

$$
K^{k^{\prime}}(t)=\sqrt{\frac{2}{\pi}} \frac{Q}{v_{0}} M\left(k^{\prime}\right) \frac{H\left[t-\sqrt{b^{2}-c^{2}}\left(h+h_{k^{\prime}}\right)+c\left(l-l_{k^{\prime}}\right)\right]}{\left[t-\sqrt{b^{2}-c^{2}}\left(h+h_{k^{\prime}}\right)+c\left(l-l_{k^{\prime}}\right)\right]^{1 / 2}} .
$$

The term with two summations comes from the contributions for waves which are diffracted by the crack tip, i.e., $k^{\prime}=0, l^{\prime}=1$ for $D R$ wave, $l^{\prime}=2$ for $D R D R$ wave; $k^{\prime}=1, l^{\prime}=1$ for 
$R R D R$ wave, $l^{\prime}=2$ for $R R D R D R ; k^{\prime}=2, l^{\prime}=1$ for $R R R R D R$ wave. The correspondent results are

$$
\begin{aligned}
K^{k^{\prime}, 1}(t) & \\
= & \sqrt{2 / \pi} \frac{Q}{4 \pi^{2}} M\left(k^{\prime}\right) \int_{0}^{t} \frac{1}{\sqrt{t-u}} \\
& \times \operatorname{Im}\left[\frac{\left(b+\eta_{k^{\prime}+2}^{+}\right) H\left[t-2 b h-\sqrt{b^{2}-c^{2}}\left(h+h_{k^{\prime}}\right)+c\left(l-l_{k^{\prime}}\right)\right]}{\left[-\cos \left(2 k^{\prime}+1\right) \phi+\sqrt{b^{2} v_{0}^{2}-1} \sin \left(2 k^{\prime}+1\right) \phi-v_{0} \eta_{k^{\prime}+2}^{+}\right]\left(b+S_{2, k^{\prime}+2}^{+}\right)^{1 / 2}}\right. \\
& \left.\frac{\partial S_{2, k^{\prime}+2}^{+}}{\partial u}\right] \mathrm{d} u,
\end{aligned}
$$

where

$$
\begin{aligned}
& l_{0}=0, \quad h_{0}=0, \\
& l_{k^{\prime}}=\sum_{m=1}^{k^{\prime}} 2 h \sin (2 m \phi), \quad h_{k^{\prime}}=\sum_{m=1}^{k^{\prime}} 2 h \cos (2 m \phi) \\
& M\left(k^{\prime}\right)=\frac{\sqrt{b^{2} v_{0}^{2}-1} \cos \left(2 k^{\prime}+1\right) \phi+\sin \left(2 k^{\prime}+1\right) \phi}{\sqrt{b^{2} v_{0}^{2}-1}\left[b-c \cos \left(2 k^{\prime}+1\right) \phi+\sqrt{b^{2}-c^{2}} \sin \left(2 k^{\prime}+1\right) \phi\right]^{1 / 2}} \\
& \eta_{k^{\prime}+2}^{+}=\frac{1}{d_{j}}\left\{-\left[t+c\left(l-l_{k^{\prime}}\right)-\sqrt{b^{2}-c^{2}}\left(h+h_{k^{\prime}}\right)\right] \cos \psi_{j}\right. \\
& \left.+i \sin \psi_{j}\left[\left(t+c\left(l-l_{k^{\prime}}\right)-\sqrt{b^{2}-c^{2}}\left(h+h_{k^{\prime}}\right)\right)^{2}-b^{2} d_{j}^{2}\right]^{1 / 2}\right\}
\end{aligned}
$$

and

$$
\begin{aligned}
K^{k^{\prime}, l^{\prime}}(t)= & -\frac{\sqrt{2 / \pi} i^{q} Q M\left(k^{\prime}\right)}{4 \pi^{2}(2 \pi i)^{l^{\prime}-1}} \int_{t^{*}}^{t} \frac{1}{\sqrt{t-u}} \int_{b^{*}}^{b_{2}} \int_{2 h b}^{b_{3}} \cdots \int_{2 h b}^{b_{l^{\prime}}} \\
& F U N K_{k^{\prime}, l^{\prime}} \mathrm{d} t_{l^{\prime}} \mathrm{d} t_{l^{\prime}-1}, \ldots, \mathrm{d} t_{2} \mathrm{~d} u
\end{aligned}
$$

where

$$
\begin{aligned}
& t^{*}=\sqrt{b^{2}-c^{2}}\left(h+h_{k^{\prime}}\right)-c\left(l-l_{k^{\prime}}\right)+2 l^{\prime} h b, \\
& b^{*}=\sqrt{b^{2}-c^{2}}\left(h+h_{k^{\prime}}\right)-c\left(l-l_{k^{\prime}}\right), \\
& b_{2}=u-2 l^{\prime} h b, \\
& b_{m+2}=u-t_{2}-t_{3}-\cdots-t_{m+1}-2\left(l^{\prime}-m\right) h b, \quad m=1,2, \ldots, l^{\prime}-2 \\
& q=0, \text { when } l^{\prime}=3,5, \ldots ; \\
& q=1, \text { when } l^{\prime}=2,4,6, \ldots,
\end{aligned}
$$




$$
\begin{aligned}
F U N K_{k^{\prime}, l^{\prime}}= & \text { Op }\left[\frac{\left(b+\eta_{k^{\prime}+l^{\prime}+1}^{+}\right) G\left(S_{2, k^{\prime}+1}^{ \pm}, \eta_{k^{\prime}+2}^{ \pm}\right) G\left(S_{2, k^{\prime}+2}^{ \pm}, \eta_{k^{\prime}+3}^{ \pm}\right)}{-\cos \left(2 k^{\prime}+1\right) \phi+\sqrt{b^{2} v_{0}^{2}-1} \sin \left(2 k^{\prime}+1\right) \phi-v_{0} \eta_{k^{\prime}+l^{\prime}+1}^{+}} \cdots\right. \\
& \cdots G\left(S_{2, k^{\prime}+l^{\prime}}^{ \pm}, \eta_{k^{\prime}+l^{\prime}+1}^{+}\right)\left( \pm \frac{\partial S_{2, k^{\prime}+1}^{ \pm}}{\partial t_{k^{\prime}+1}}\right) \cdots \\
& \left.\cdots\left( \pm \frac{\partial S_{2, k^{\prime}+l^{\prime}}^{ \pm}}{\partial t_{k^{\prime}+l^{\prime}}}\right)\left(\frac{\partial S_{2, k^{\prime}+l^{\prime}+1}^{+}}{\partial t_{k^{\prime}+l^{\prime}+1}}\right)\right]
\end{aligned}
$$

in which the operator 'Op' is ' $\mathrm{Re}^{\prime}$ (or 'Im') if $l^{\prime}$ is even (or odd) and

$$
\begin{aligned}
& \eta_{k^{\prime}+2}^{ \pm}=-\frac{t_{k^{\prime}+2} \sin \phi}{2 h} \pm i \frac{\cos \phi}{2 h}\left(t_{k^{\prime}+2}^{2}-4 b^{2} h^{2}\right)^{1 / 2}, \\
& S_{2, k^{\prime}+v^{\prime}+1}^{ \pm}=\frac{t_{k^{\prime}+v^{\prime}+1} \sin \phi}{2 h} \pm i \frac{\cos \phi}{2 h}\left(t_{k^{\prime}+v^{\prime}+1}^{2}-4 b^{2} h^{2}\right)^{1 / 2}, \quad v^{\prime}=1,2,3, \ldots, l^{\prime}, \\
& \eta_{k^{\prime}+v^{\prime}+2}^{+}=-\frac{t_{k^{\prime}+l^{\prime}+p+2} \sin \phi}{2 h} \pm i \frac{\cos \phi}{2 h}\left(t_{k^{\prime}+v^{\prime}+2}^{2}-4 b^{2} h^{2}\right)^{1 / 2}, \quad v^{\prime}=1,2,3, \ldots, l^{\prime} .
\end{aligned}
$$

The number of reflected waves (i.e. $n$ and $n^{\prime}$ ) which will pass the crack tip and induce diffracted waves is dependent on the location and the inclined angle $\phi$ of the crack, the speed of the moving load and the position where the dynamic point loading is initially applied. The results are expressed as follows

If $\phi_{2 j+1}<\pi / 2<\phi_{2 j-1}, \quad$ then $n=j$,

where

$$
\begin{aligned}
& \phi_{2 j+1}=\cos ^{-1}\left(\frac{l_{2 j+1}}{R_{2 j+1}}\right) \\
& R_{2 j+1}=\left(l_{2 j+1}^{2}+h_{2 j+1}^{2}\right)^{1 / 2}, \\
& l_{2 j+1}=l \cos 2(j+1) \phi+h \sin 2(j+1) \phi+\sum_{p=1}^{n} 2 h \sin (2 p \phi), \\
& h_{2 j+1}=l \sin 2(j+1) \phi-h \cos 2(j+1) \phi-\sum_{p=1}^{n} 2 h \cos (2 p \phi) .
\end{aligned}
$$

If $\pi-\cos ^{-1}\left(\frac{l_{2 k}}{R_{2 k}}\right)<\cos ^{-1}\left(\frac{c}{v_{0}}\right)+2 k \phi<\frac{1}{2} \pi$ and $\cos ^{-1}\left(\frac{c}{v_{0}}\right)+(2 k+2) \phi \geqslant \frac{1}{2} \pi$, then $n^{\prime}=k$, where

$$
\begin{aligned}
& l_{0}=l, \quad h_{0}=h \\
& l_{2 k}=l \cos (2 k \phi)+h \sin (2 k \phi)+\sum_{m=0}^{k-1} 2 h \sin 2 m \phi, \quad k=1,2,3, \ldots \\
& h_{2 k}=-l \sin (2 k \phi)+h \cos (2 k \phi)+\sum_{m=0}^{k-1} 2 h \cos 2 m \phi, \quad k=1,2,3, \ldots \\
& R_{2 k}=\left(l_{2 k}^{2}+h_{2 k}^{2}\right)^{1 / 2}
\end{aligned}
$$




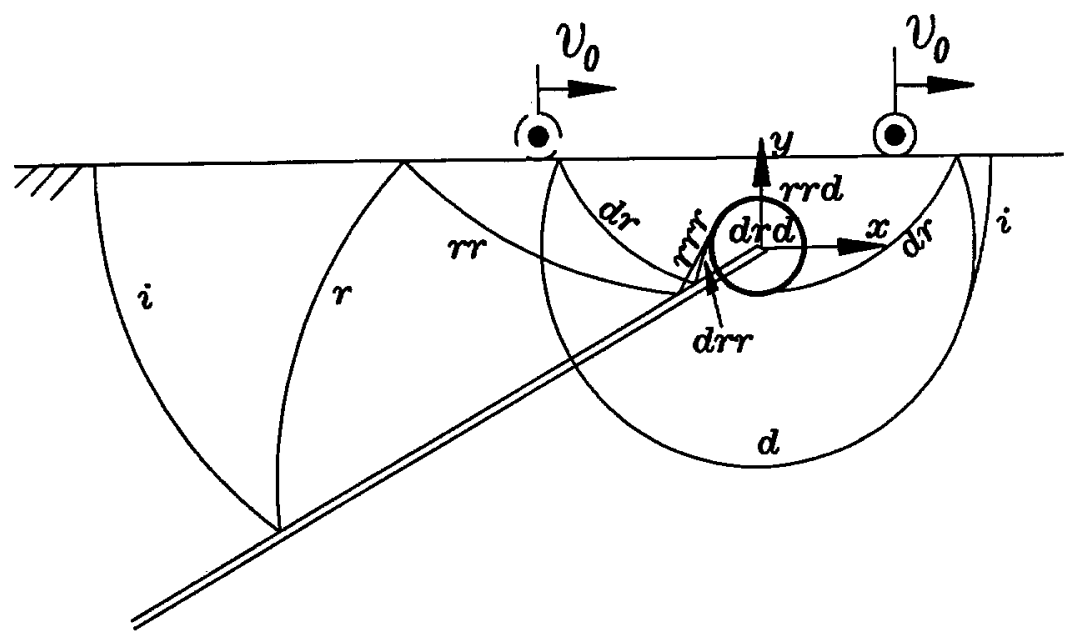

Figure 4. Wave fronts for a short time period after the dynamic loading is applied on the half-space for the subsonic case.

\section{Numerical results of stress intensity factors}

The geometric configuration considered in this study is an inclined semi-infinite crack located under the surface of a half-plane. The incident wave (for subsonic case) generated by the dynamic antiplane loading will be diffracted from the crack tip and reflected from the crack surface as $d$ and $r$ waves, which will be reflected from the half-plane as a $d r$ and $r r$ wave and interact with the inclined crack again at a later time. These waves will reflect back and forth between the half-plane and the crack, which will generate an infinite number of reflected and diffracted waves. The wave fronts for incident, reflected and diffracted waves (subsonic case) for a short time period are expressed in Figure 4.

In the previous section, the exact transient closed form solution of the stress intensity factor has been determined. The transient response of the stress intensity factor will be investigated numerically here for a point dynamic loading with a Heaviside function $H(t)$ time dependence initially applied at $(-25,10)$ and moving with a constant speed in the positive $x$-direction. The applied loading is located at the left hand side of the crack tip and the inclined angle $\phi$ of the crack is chosen to be $30^{\circ}$. The transient responses of dynamic stress intensity factors for both the subsonic and supersonic are shown in Figure 5. The time has been normalized by dividing $b R_{0}$, where $R_{0}$ is the distance from the location of point loading initially applied to the crack tip. The corresponding static value is also indicated in the figures. The normalized arrival times for the first few wave fronts at the crack tip are 1 for the incident $i$ wave, 1.738 for the $r r$ wave, 1.742 for the $d r$ wave and 2.48 for the $r r d r$ wave. The history of stress intensity factor for the subsonic case shows a finite jump as the $i$ wave and $r r$ wave have arrived at the crack tip. The transient solution of the stress intensity factor for the case $v_{0}=0$ will tend toward the corresponding static value after the second diffracted wave has passed the crack tip.

Finally, we consider the case where the crack is parallel to the half plane surface, i.e. $\phi=0$. For the numerical investigation, we consider a dynamic point loading with a Heaviside function time dependence initially applied at $(-1,1)$ (or $(0.5,2))$ which is at the left (or right) 


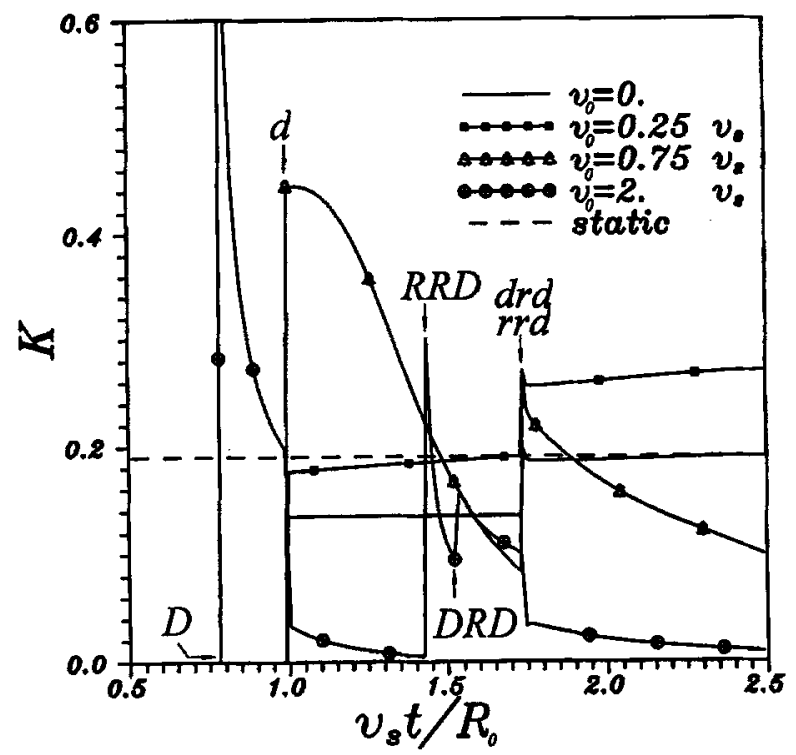

Figure 5. Transient response of the dynamic stress intensity factor due to the impact loading applied at $(-25,10)$ for the inclined angle $\phi=30^{\circ}$.

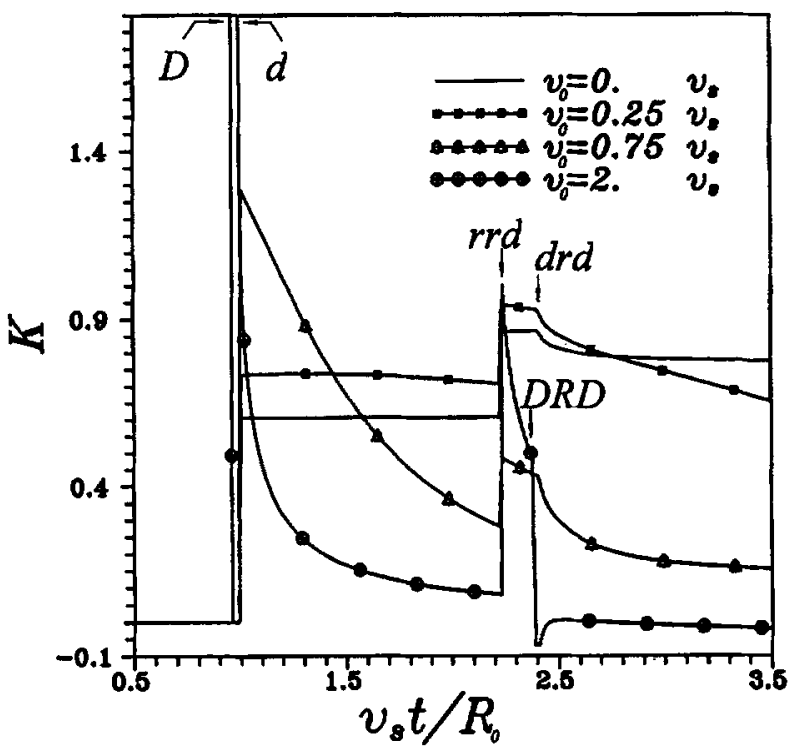

Figure 6. Transient response of the dynamic stress intensity factor due to the impact loading applied at $(-1,1)$ for the crack parallel to the half-space.

hand side of the crack tip. The transient history of the dynamic stress intensity factor is shown in Figures 6 and 7, both for subsonic and supersonic cases.

\section{Conclusions}

Most of the problems which have been studied in the development of fracture mechanics are quasi-static. Because of loading conditions and material properties, numerous problems 


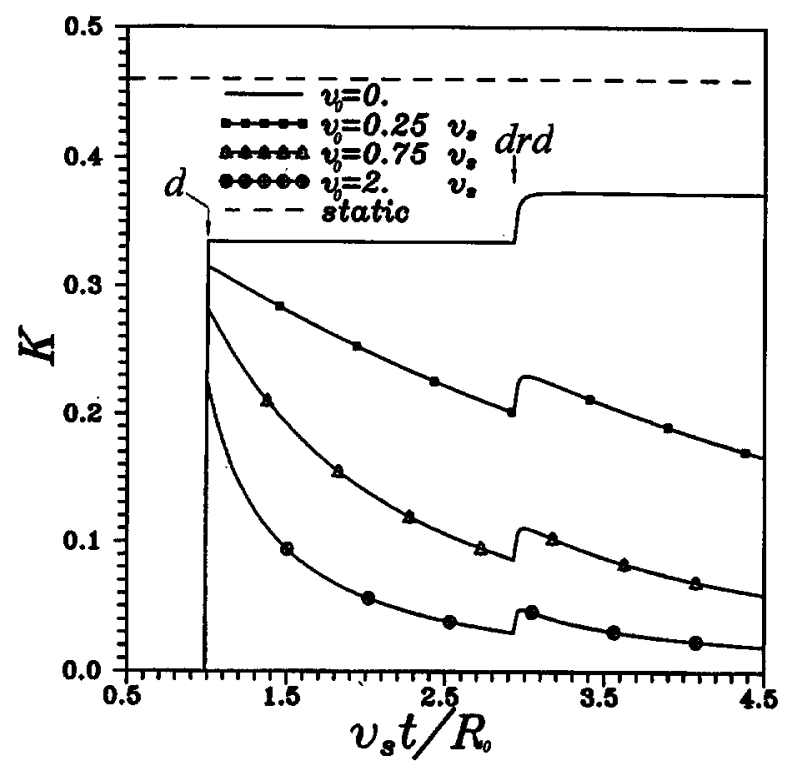

Figure 7. Transient response of the dynamic stress intensity factor due to the impact loading applied at $(0.5,2)$ for the crack parallel to the half-space.

have existed for which the assumption that the deformation is quasi-static is invalid and the inertia of the material must be taken into account. The propagation of stress waves through an unbounded medium is not a difficult subject. However, if boundaries are introduced, infinitely many reflected and diffracted waves will be generated from boundaries, making the problem much more complicated. In dynamic fracture analysis, the interest is in the determination of the stress field near the crack tip in order to study the phenomenon of crack propagation under dynamic loading. This study provides a useful methodology and results for a cracked body subjected to dynamic moving loadings.

The transient response of a half-space containing a subsurface inclined crack has been considered here to gain insight into understanding the interaction of stress waves generated by dynamic moving loading with material defects. This problem contains a characteristic length and is solved by superposition of proposed fundamental solutions in the Laplace transform domain. This new methodology is shown to be both powerful and efficient in solving more difficult problems. The exact transient solutions of dynamic stress intensity factor for a long period of time are obtained in this study. The closed form transient solutions for both subsonic and supersonic cases are expressed in a very simple formulation which accounts for all the contributions coming from incident, reflected and diffracted waves. The numerical calculations are also investigated for both subsonic and supersonic cases. For the special case of the moving speed of the dynamic loading equal to zero, the transient result will approach the correspondent static value after the first few waves have passed the crack tip. The solution of dynamic stress intensity factor obtained in this study can be used for analyzing the condition for the unstable crack propagation under dynamic moving loading. 


\section{Acknowledgments}

The research support of the National Science Council, Republic of China, through Grant NSC 83-0401-E002-115 at National Taiwan University is gratefully acknowledged.

\section{References}

1. R.G. Payton, Quarterly of Applied Mathematics 21 (1964) 299-313.

2. D.C. Grakenheimer and J. Miklowitz, Journal of Applied Mechanics 36 (1969) 505-515.

3. B. Noble, The Wiener-Hopf Technique. Pergamon Press, Oxford (1958).

4. A.T. de Hoop, 'Representation theorems for the displacement in an elastic solid and their application to elastodynamic diffraction theory', Doctoral dissertation, Technische Hogeschool, Delft (1958).

5. L.B. Freund, International Journal of Engineering Science 12 (1974) 179-189.

6. L.M. Brock, International Journal of Solids and Structures 18 (1982) 467-477.

7. L.M. Brock, Journal of Elasticity 14 (1984) 415-424.

8. L.M. Brock, M. Jolles and M. Schroedl, Journal of Applied Mechanics 52 (1985) 287-290.

9. C.C. Ma and Y.C. Hou, International Journal of Engineering Science 28 (1990) 1321-1329.

10. C.C. Ma and Y.C. Hou, Journal of Applied Mechanics 58 (1991) 703-709.

11. C.H. Tsai and C.C. Ma, Journal of Applied Mechanics 59 (1992) 804-811.

12. C.C. Ma and S.K. Chen, Wave Motion 17 (1993) 161-171.

13. L.M. Brock, Quarterly of Applied Mathematics XLIV (1986) 265-275.

14. X.P. Li and C.T. Liu, International Journal of Fracture 69 (1995) 319-339.

15. J.D. Achenbach and R.J. Brind, Journal of Sound and Vibration 76 (1981) 43-56.

16. R.J. Brind and J.D. Achenbach, Journal of Sound and Vibration 76 (1981) 555-563.

17. C.C. Ma and S.K. Chen, ASME Journal of Applied Mechanics 61 (1994) 649-655.

18. C.C. Ma and L.R. Hwang, International Journal of Solids and Structures (1996) in press.

19. L. Cagniard, Reflection and Refractions of Progressive Seismic Waves, McGraw Hill, New York (1958). 